\title{
Mucinous Appendiceal Tumor Presenting as Perforated Appendicitis
}

\author{
Kathleen B. To, ${ }^{1}$ Scott R. Owens, ${ }^{2}$ and Lena M. Napolitano ${ }^{1}$
}

\begin{abstract}
A
PREVIOUSLY HEALTHY 60-year-old male presented with a 1-wk history of abdominal pain localized to the right lower quadrant, fever, and loose stools. He reported nausea, anorexia and a 5-lb weight loss over the preceding week. There was mild right lower quadrant abdominal tenderness
\end{abstract}

on examination. Computed tomography (CT) of the abdomen and pelvis showed a thick-walled, inverted C-shaped structure with associated inflammatory changes in the right lower quadrant (Fig. 1). The patient responded to initial non-surgical management with intravenous anti-microbial
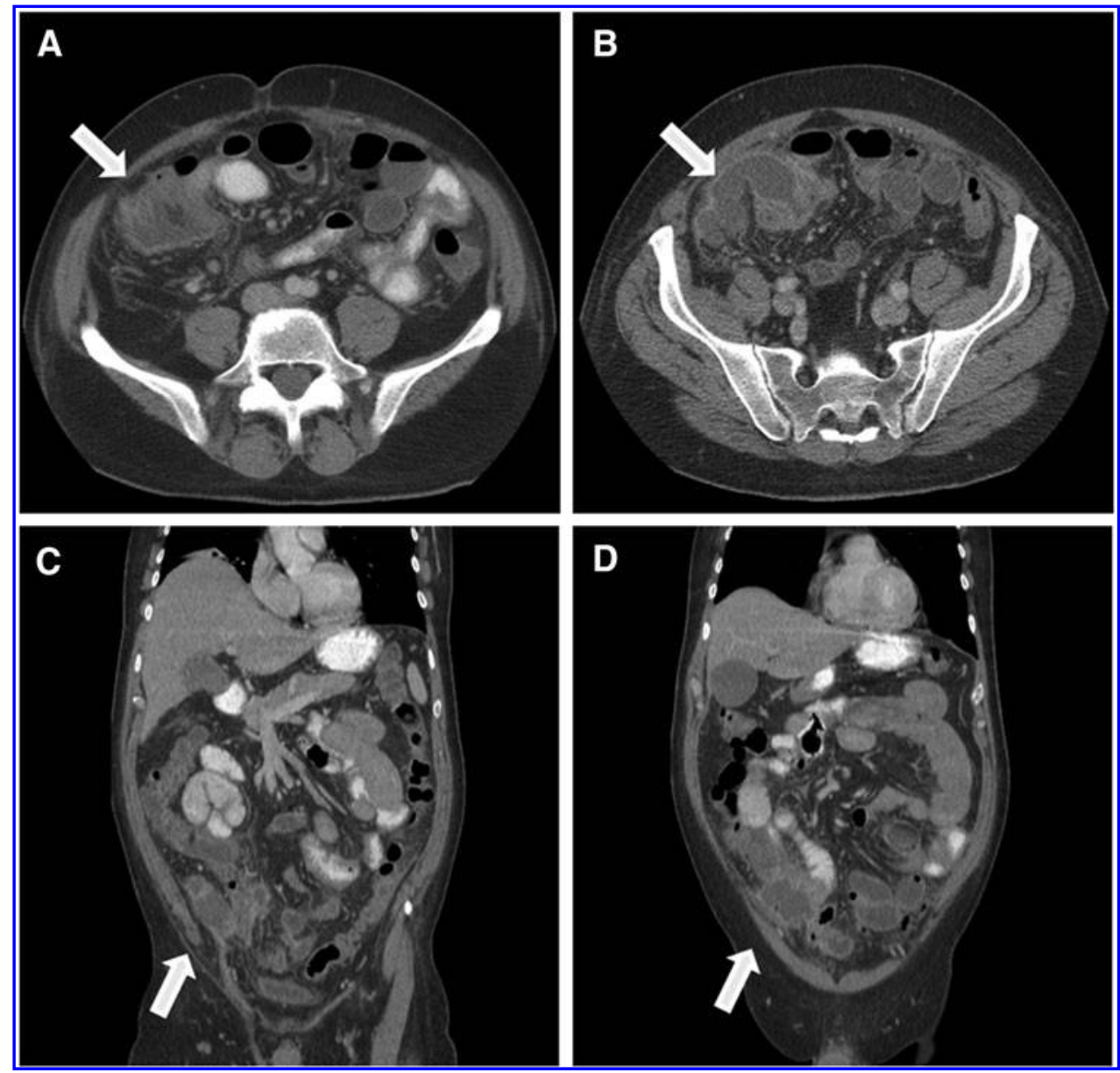

FIG. 1. Computed tomography image of the abdomen and pelvis demonstrating right lower quadrant inflammatory phlegmon (arrow). (A, B) Axial views. (C, D) Coronal views.

\footnotetext{
${ }^{1}$ Division of Acute Care Surgery, Department of Surgery, ${ }^{2}$ Division of Anatomic Pathology, Department of Pathology, University of Michigan Hospital and Health Systems, Ann Arbor, Michigan.
} 
therapy (piperacillin/tazobactam) for the acute inflammatory process, presumed to be either from perforated appendicitis or a mucinous appendiceal tumor. Subsequent surgical intervention was appendectomy; operative findings confirmed no mucin in the peritoneal cavity. Final pathology showed a low-grade appendiceal mucinous cystic neoplasm with acellular extra-appendiceal mucin and surrounding fibrousinflammatory tissue, but no extra-appendiceal epithelial cells and no evidence of carcinoma (Fig. 2). Recovery was uncomplicated and the patient was referred to medical oncology for long-term follow-up.

Mucinous neoplasm of the appendix is uncommon, with a reported incidence of $0.29-0.4 \%$ of all appendectomies [1]. Rokitansky first described a mucocele of the appendix in 1842. Variability of terminology describing the mucinous degeneration of these rare appendiceal tumors has led to much controversy over their classification. One of the reasons for the controversy lies in the association of appendiceal epithelial tumors with pseudomyxoma peritoneii (PP), a term coined by Werth in 1884 to describe a low-grade neoplastic process that results in mucinous aggregates in the

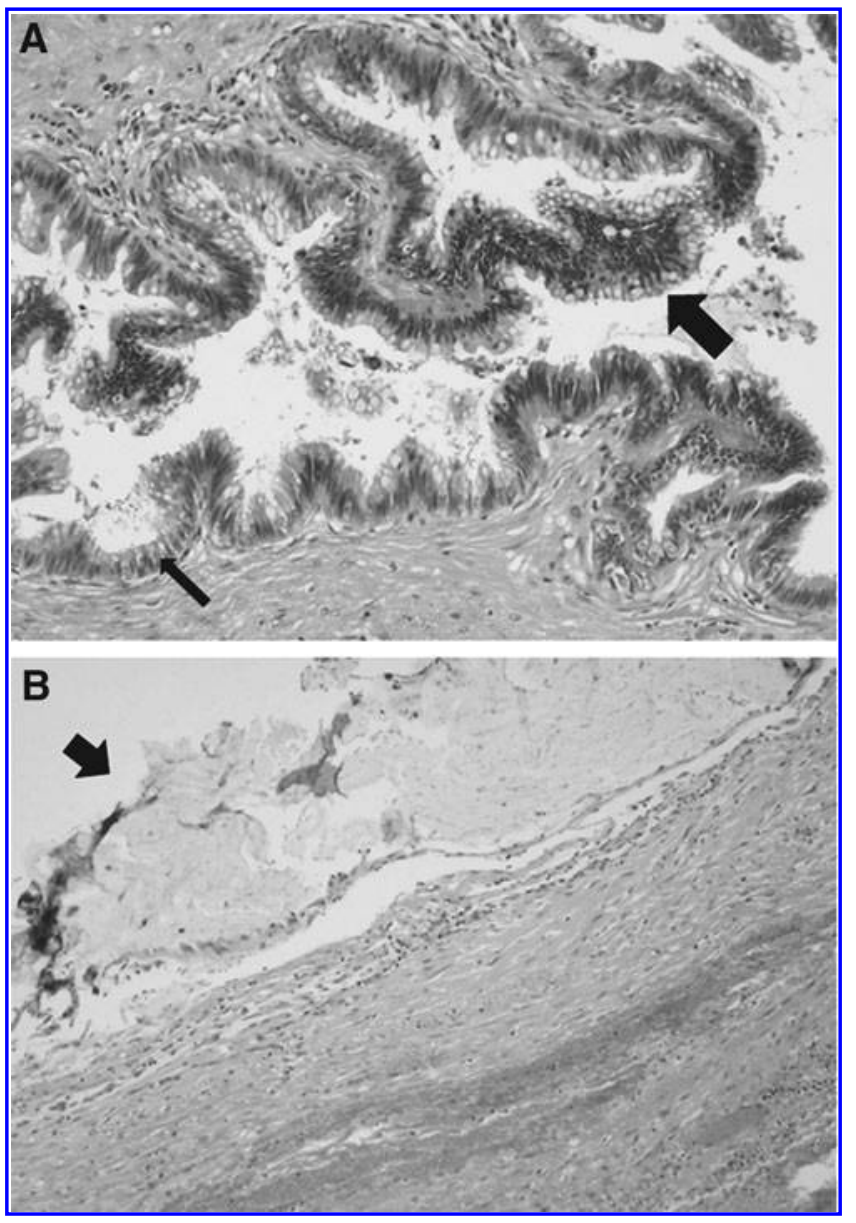

FIG. 2. Hematoxylin and eosin stain. (A) Normal appendiceal mucosal cells (thin arrow) and low-grade appendiceal mucinous neoplasm (LAMN, thick arrow) with acellular mucin in the appendiceal lumen. (B) Acellular mucin in the appendiceal lumen (arrow). peritoneal cavity and is associated with substantial morbidity and mortality [2]. Misdraji et al. [3] proposed the terms low-grade appendiceal mucinous neoplasm (LAMN) and mucinous adenocarcinomas (MACA) to characterize better the prognostic importance of this complex spectrum of disease. LAMNs have also been reported under other names: Villous adenoma, cystadenoma, mucinous tumor of uncertain malignant potential, borderline tumor of the appendix, and mucocele. In their review of 107 specimens diagnosed as appendiceal mucinous neoplasms, Misdraji et al. [3] found that LAMNs confined to the appendix had no recurrence (median follow-up, 6 mo); LAMNs with extra-appendiceal spread had similar 5- and 10-y survival rates to MACA ( $86 \%$ versus $90 \%$, and $45 \%$ versus $44 \%$, respectively).

Almost 50\% of mucinous neoplasms of the appendix are not diagnosed pre-operatively and up to $25 \%$ are asymptomatic. Often the diagnosis of LAMN is made at the time of surgery for right lower quadrant pain. However, certain features may be noted on CT, such as the presence of a cystic mass with low attenuation or irregular wall thickening, especially in the absence of peri-appendiceal inflammation. Ultrasound may show an elongated, echo-poor cystic mass with liquid and viscous content; calcifications may sometimes be present [4]. Magnetic resonance imaging (MRI) may demonstrate a cystic mass with low signal intensity on T1-weighted images and higher signal intensity on T2weighted images because of the high protein content of the mucin. Endoscopy may demonstrate a "volcano sign," a smooth mound with normal overlying mucosa surrounding the appendiceal opening.

The treatment for patients with LAMN often depends on the presenting symptoms and intraoperative findings. LAMN confined to the appendix with no extra-appendiceal involvement can be treated with appendectomy. If the diagnosis of a mucinous appendiceal tumor is suspected preoperatively, a thorough search for extra-appendiceal spread is warranted, and intra-operative precautions to avoid mucin spillage is key. However, given the natural history of LAMN, in the absence of malignant disease or involvement of the appendiceal base, more extensive surgical resection such as a formal right hemicolectomy is unlikely to improve patient outcome [2,3]. Nevertheless, on the basis of findings in a population-based study of more than 167,000 appendectomies spanning a $10-y$ period, Smeenk et al. [5] recommend a minimum 5-y follow-up (physical examination, tumor markers [carcinoembryonic antigen and cancer antigen 19-9], and annual CT scan) in patients with any mucinous appendiceal epithelial neoplasms to evaluate for the development of PP.

\section{References}

1. Karakaya K, Barut F, Emre AU, et al. Appendiceal mucocele: Case reports and review of current literature. World J Gastroenterol 2008;14:2280-2283.

2. Misdraji J. Appendiceal mucinous neoplasms: Controversial issues. Arch Pathol Lab Med 2010;134:864870 .

3. Misdraji J, Yantiss RK, Graeme-Cook FM, et al. Appendiceal mucinous neoplasms: A clinicopathologic analysis of 107 cases. Am J Surg Pathol 2003;27:1089-1103. 
4. Madwed D, Mindelzun R, Jeffrey RB Jr. Mucocele of the appendix: Imaging findings. AJR Am J Roentgenol 1992; 159:69-72.

5. Smeenk RM, van Velthuysen ML, Verwaal VJ, Zoetmulder FA. Appendiceal neoplasms and pseudomyxoma peritonei: A population based study. Eur J Surg Oncol 2008;34: 196-201.
Address correspondence to: Dr. Kathleen B. To Division of Acute Care Surgery Department of Surgery University of Michigan Hospital and Health Systems 1500 East Medical Center Dr., UH 1C-340 Ann Arbor, MI 48109-5033

E-mail:kato@umich.edu 